\title{
LINEARIZAÇÃO DOS COEFICIENTES DE REFLEXÃO DE ONDAS qP EM MEIOS ANISOTRÓPICOS
}

\author{
Ellen N. S. Gomes ${ }^{1}$, João S. Protázio ${ }^{1}$, Jessé C. Costa ${ }^{1}$ \& Ivan A. Simões \\ Filho $^{2}$
}

Received September 15, 2000 / Accepted March 25, 2001

\begin{abstract}
Os coeficientes de reflexão de onda qP em uma interface plana que separa dois meios anisotrópicos dependem dos parâmetros elásticos dos meios envolvidos de maneira não linear. Aproximações lineares para a refletividade de ondas qP pressupondo fraco contraste de impedância e fraca anisotropia levam a uma forma simples para se fazer à análise de AVO/AVD. Neste trabalho a solução das equações de Zoeppritz é rescrita explicitamente em função de matrizes de impedância e polarização. Além disso, utilizando-se esta abordagem, é apresentada uma metodologia geral mais simples para se obter as formas linearizadas. Estas formas linearizadas para os coeficientes de reflexão da onda $\mathrm{qP}$ e das ondas convertidas para uma onda $\mathrm{qP}$ incidente apresentam resultados muito próximos da formulação exata para ângulos de incidência menores que $30^{\circ}$ considerando-se um contraste de impedância moderado e anisotropia em limites geologicamente aceitáveis.
\end{abstract}

Palavras-chave : Anisotropia; AVO/AVD.

LINEARIZED REFLECTION COEFFICIENTS FOR qP WAVES IN ANISOTROPIC MEDIA - The reflection coefficients at a planar interface separating two anisotropic media have a nonlinear dependence on the elastic parameters and densities of both media. Linear approximations on the elastic parameters for the $q P$ wave reflectivity are more convenient for AVO/AVD analysis. We present the solution of the Zoeppritz equations in terms of impedance and polarization matrices. Using this approach and assuming weak impedance contrast and weak anisotropy, a simple derivation of linearized approximations for $q P$ the reflectivity is presented for general anisotropy. The linear approximations of reflection coefficients, $q P$ and converted waves, for $q P$ incidence are very close to the exact results for incidence angles up to 30 degrees considering moderate impedance contrast and anisotropy.

Key words: Anisotropy; $A V O / A V D$. 


\section{INTRODUÇÃO}

As propriedades elásticas das rochas na subsuperfície podem ser estimadas através do estudo das amplitudes das ondas refletidas utilizando-se a análise da amplitude versus afastamento (AVO) e amplitude versus direção (AVD). Essas análises têm sido importantes no prognóstico de contrastes litológicos ou de conteúdo de fluidos das formações. Em meios com fraturamento pode-se, por exemplo, determinar a orientação de planos de fratura que em geral indicam a direção de maior permeabilidade que é essencial à caracterização de reservatórios de hidrocarbonetos.

Os primeiros trabalhos sobre o assunto levaram em consideração apenas meios acústicos ou elásticos isotrópicos (Ostrander, 1984). Novas técnicas de aquisição de dados em 3-C (três componentes) e o comportamento efetivamente anisotrópico de meios fraturados (Schoenberg \& Douma, 1988) e finamente estratificados (Postma, 1955) em regime quase estático estimularam a incorporação de anisotropia na análise de AVO/AVD. Modelos interpretativos que admitem anisotropia apresentam expressões analíticas das amplitudes que dependem de forma não linear dos parâmetros elásticos do meio o que torna a análise destas expressões complexa. Devido a esta dificuldade, formas linearizadas para as amplitudes têm sido utilizadas.

Várias abordagens têm sido propostas para simplificar a expressão exata do coeficiente de reflexão de uma onda qP supondo uma onda qP incidente. Para meios fracamente anisotrópicos com alto grau de simetria e baixo contraste, os principais trabalhos foram de Thomsen (1986; 1993) e Banik (1987). Uma aproximação foi apresentada por Zillmer et al. (1997) considerando meios fracamente anisotrópicos e contrastes arbitrários. Entretanto, essa forma aproximada é ainda muito complexa. Vavrycuk \& Psencik (1998) a particularizaram considerando pequenos contrastes entre as propriedades elásticas do meio obtendo uma fórmula mais simples tanto para o coeficiente de reflexão como para o coeficiente de transmissão de uma onda qP considerando uma onda qP incidente.

Neste artigo faz-se uma extensão do trabalho de Schoenberg \& Protázio (1992), no qual uma formulação matricial para o cálculo exato dos coeficientes de reflexão para meios anisotrópicos com pelo menos um plano de simetria foi apresentado. Estende-se esta formulação determinando-se os coeficientes de reflexão e transmissão exatos entre meios anisotrópicos arbitrários. Além disso, a partir desta formulação, em que as equações de Zoeppritz, são escritas de forma particionada, é obtida uma forma linearizada para os coeficientes de reflexão da onda qP e de suas convertidas considerando uma onda incidente qP. A grande vantagem deste método é a simplificação da obtenção das linearizações. Faz-se também uma avaliação destas aproximações a partir de dados sintéticos mostrando-se que as fórmulas linearizadas produzem resultados muito próximos daqueles obtidos com a formulação exata para incidências de até $30^{\circ}$ que é a faixa usada na prospeção sísmica.

\section{METODOLOGIA}

Neste trabalho, as matrizes são representadas por letras maiúsculas e em negrito e os vetores são representados por letras minúsculas e em negrito, utiliza-se a notação indicial e a convenção da soma (Aki \& Richards, 1980) em todo o texto e as exceções a estas regras serão indicadas explicitamente. Além disso, será utilizada a notação $\mathrm{P}, \mathrm{S}$ e T para as ondas $\mathrm{qP}, \mathrm{qS}_{1}$ e $\mathrm{qS}_{2}$ (Helbig, 1994)

Sejam dois meios anisotrópicos quaisquer separados por uma interface plana $\mathrm{x}_{3}=0$ com a incidência ocorrendo no meio superior e a transmissão ocorrendo no meio subjacente. Como estamos considerando que as medidas serão feitas longe da fonte e para intervalos de tempo muito pequenos, uma boa aproximação para o espalhamento é considerar que as ondas incidentes, refletidas e transmitidas são ondas planas cujo campo de deslocamento é descrito por :

$$
\mathbf{u}_{\alpha}(\mathbf{x}, t)=\mathrm{A}_{\alpha} \mathbf{n}_{\alpha} \exp \left[i \omega\left(\mathbf{s}_{\alpha} . \mathbf{x}-t\right)\right],
$$

sendo $\mathbf{u}$ o vetor de deslocamento, $\mathbf{n}_{\alpha}$ o vetor polarização, $\mathrm{A}_{\alpha}$ a amplitude da onda, $\mathbf{s}_{\alpha} \mathrm{o}$ vetor vagarosidade, $\omega$ é a freqüência e x é o vetor posição 
espacial (Musgrave, 1970). O subscrito $\alpha$ refere-se às ondas $\mathrm{P}, \mathrm{S}$ e $\mathrm{T}$, assim classificadas de acordo com sua velocidade de propagação.

Utilizando a lei de Hooke generalizada e considerando que na interface o campo de deslocamento e a tração são contínuos, os coeficientes de reflexão e transmissão das ondas espalhadas são determinados pelo sistema matricial:

$$
\left\{\begin{array}{l}
\mathbf{N}_{i} \mathbf{i}+\mathbf{N}_{r} \mathbf{r}=\mathbf{N}_{t} \mathbf{t} \\
\mathbf{Z}_{i} \mathbf{i}+\mathbf{Z}_{r} \mathbf{r}=\mathbf{Z}_{t} \mathbf{t},
\end{array}\right.
$$

em que i corresponde à amplitude da onda incidente, $\mathrm{r}$ à amplitude da onda refletida e t à amplitude da onda transmitida. As matrizes $\mathbf{N}_{k}$ e $\mathbf{Z}_{k}$ correspondem às matrizes de polarização e impedância da onda incidente $(\mathrm{k}=\mathrm{i})$, refletida $(\mathrm{k}=\mathrm{r})$ e transmitida $(\mathrm{k}=\mathrm{t})$ respectivamente, as componentes destas matrizes são obtidas por $\mathrm{N}_{\mathrm{m} \alpha}=\mathrm{n}_{\mathrm{m} \alpha}$, $\mathrm{Z}_{\mathrm{m} \alpha}=\mathrm{c}_{3 \mathrm{mjl}} \mathrm{s}_{1 \alpha} \mathrm{n}_{\mathrm{j} \alpha}$ e $\mathrm{c}_{3 \mathrm{jkl}}$ representa as componentes do tensor elástico do meio. Neste trabalho as componentes do tensor elástico serão representadas utilizando a notação reduzida (Helbig, 1994) em que $c_{m j k 1}$ será substituído por $C_{p q}$.

Explicitamente os coeficientes r e t são dados por :

$$
\begin{aligned}
& \mathbf{r} \equiv \mathbf{R} \mathbf{i}=\left(\mathbf{Z}_{r}-\mathbf{Z}_{t} \mathbf{N}_{t}^{-1} \mathbf{N}_{r}\right)^{-1}\left(\mathbf{Z}_{i}-\mathbf{Z}_{t} \mathbf{N}_{t}^{-1} \mathbf{N}_{i}\right) \mathbf{i} \\
& \mathbf{t} \equiv \mathbf{T} \boldsymbol{i}=-\left(\mathbf{Z}_{t}-\mathbf{Z}_{r} \mathbf{N}_{r}^{-1} \mathbf{N}_{t}\right)^{-1}\left(\mathbf{Z}_{i}-\mathbf{Z}_{r} \mathbf{N}_{r}^{-1} \mathbf{N}_{i}\right) \mathbf{i} .
\end{aligned}
$$

As fórmulas (2) e (3) generalizam para meios anisotrópicos arbitrários os resultados obtidos por Schoenberg \& Protázio (1992) para o caso de meios com pelo menos um plano horizontal de simetria especular.

A partição do sistema de equações de Zoepprittz (2) através das matrizes de impedância e polarização permite se obter uma solução explicita para as matrizes de reflexão e transmissão o que não é possível com a abordagem usual (Vavrycuk \& Psencik, 1998). As matrizes R e T contêm todas as informações sobre as amplitudes envolvidas no espalhamento.
As amplitudes das ondas incidente, refletida e transmitidas são representados pelos vetores $\mathbf{i}=i_{\alpha}$, $\mathbf{r}=r_{\alpha} \mathrm{e} \mathbf{t}=t_{\alpha}$, respectivamente. Os coeficientes de reflexão de uma onda incidente $\mathrm{P}, \mathrm{R}_{\mathrm{PP}}, \mathrm{R}_{\mathrm{SP}}$ e $\mathrm{R}_{\mathrm{TP}}$ são obtidos de (3) em que:

$$
\mathbf{R}=\left[R_{\alpha \beta}\right]=\left[\begin{array}{lll}
R_{P P} & R_{P S} & R_{P T} \\
R_{S P} & R_{S T} & R_{S T} \\
R_{T P} & R_{T S} & R_{T T}
\end{array}\right] .
$$

Um dos possíveis problemas nesta formulação é que nas direções onde ocorre as singularidades não se pode determinar as matrizes de polarização (representadas por $\mathbf{N}$ ). Entretanto pode-se sempre escolher vetores de polarização linearmente independentes de tal forma que se possa determinar as matrizes de polarização inversíveis e desta forma o sistema (2) terá solução não trivial.

Na formulação exata dos coeficientes de reflexão os parâmetros elásticos dos meios aparecem de forma não linear o que torna a análise das propriedades destes meios a partir de AVO/AVD bastante complexa. Uma alternativa para simplificar a análise é linearizar estes coeficientes. Neste trabalho a linearização dos coeficientes $\mathrm{R}_{\mathrm{PP}}, \mathrm{R}_{\mathrm{SP}}$ e $\mathrm{R}_{\mathrm{TP}}$ é feita em duas etapas. Primeiramente, tomam-se pequenas perturbações dos parâmetros elásticos $\mathrm{C}_{33}$ e $\mathrm{C}_{55}$ em torno de um meio de referência isotrópico homogêneo.

Assim, sendo $\rho^{(\gamma)}$ e $\mathrm{C}_{\mathrm{mj}}^{(\gamma)}$ a densidade e os parâmetros elásticos dos meios incidente $(\gamma=1)$ e subjacente $(\gamma=2)$, definem-se:

$$
\begin{gathered}
\alpha^{(\gamma)}=\sqrt{C_{33}^{(\gamma)} / \rho^{(\gamma)}}, \\
\beta^{(\gamma)}=\sqrt{C_{55}^{(\gamma)} / \rho^{(\gamma)}},
\end{gathered}
$$

como as velocidades do meio isotrópico superior (onde ocorre a incidência) e do meio subjacente (onde ocorre a transmissão). A densidade e as velocidades do meio isotrópico de referência são então dadas por: 


$$
\begin{gathered}
\rho=\frac{\left(\rho^{(1)}+\rho^{(2)}\right)}{2}, \alpha=\frac{\left(\alpha^{(1)}+\alpha^{(2)}\right)}{2} \mathrm{e} \\
\beta=\frac{\left(\beta^{(1)}+\beta^{(2)}\right)}{2} .
\end{gathered}
$$

A seguir os outros parâmetros elásticos dos meios isotrópicos resultantes da primeira perturbação são também perturbados com $\mathrm{C}_{33}$ e $\mathrm{C}_{55}$ mantidos agora constantes.

Desta forma têm-se meios que respondem como meios fracamente anisotrópicos.

Perturbando o sistema linear (2), as fórmulas linearizadas dos coeficientes são então dadas pela resolução do sistema:

$$
\begin{array}{r}
\left(\widetilde{\mathbf{Z}}_{r}-\widetilde{\mathbf{Z}}_{t} \widetilde{\mathbf{N}}_{t}^{-1} \widetilde{\mathbf{N}}_{r}\right) \delta \mathbf{R}=2\left(\Delta \mathbf{Z}-\widetilde{\mathbf{Z}}_{t} \widetilde{\mathbf{N}}_{t}^{-1} \Delta \mathbf{N}\right) \\
\left(\widetilde{\mathbf{Z}}_{t}-\widetilde{\mathbf{Z}}_{r} \widetilde{\mathbf{N}}_{r}^{-1} \widetilde{\mathbf{N}}_{t}\right) \delta \mathbf{T}=-2\left(\Delta \mathbf{Z}-\widetilde{\mathbf{Z}}_{r} \widetilde{\mathbf{N}}_{r}^{-1} \Delta \mathbf{N}\right)
\end{array}
$$

As matrizes $\widetilde{\mathbf{Z}}_{k}$ e $\widetilde{\mathbf{N}}_{k}$, correspondem às matrizes de impedância e de polarização associadas à reflexão $(\mathrm{k}=\mathrm{r})$ e transmissão $(\mathrm{k}=\mathrm{t})$ do meio isotrópico de referência. Os contrastes médios entre as matrizes de polarização e de impedância dos meios incidente e subjacente são dados pelas matrizes $\Delta \mathbf{N}=\left(\delta \mathbf{N}_{t}-\delta \mathbf{N}_{i}\right) / 2$ e $\Delta \mathbf{Z}=\left(\delta \mathbf{Z}_{t}-\delta \mathbf{Z}_{i}\right) / 2$, respectivamente. As matrizes de reflexão e transmissão perturbadas $\delta \mathrm{R}$ e $\delta \mathrm{T}$ são dadas por :

$$
\begin{gathered}
\delta \mathbf{R}=\left[\begin{array}{lll}
\delta R_{P P} & \delta R_{P S} & \delta R_{P T} \\
\delta R_{S P} & \delta R_{S T} & \delta R_{S T} \\
\delta R_{T P} & \delta R_{T S} & \delta R_{T T}
\end{array}\right], \\
\delta \mathbf{T}=\left[\begin{array}{lll}
\delta T_{P P} & \delta T_{P S} & \delta T_{P T} \\
\delta T_{S P} & \delta T_{S T} & \delta T_{S T} \\
\delta T_{T P} & \delta T_{T S} & \delta T_{T T}
\end{array}\right] .
\end{gathered}
$$

Na determinação da aproximação linear para os coeficientes de reflexão para incidência $\mathrm{P}, \delta \mathrm{R}_{\mathrm{PP}}$, $\delta \mathrm{R}_{\mathrm{SP}}$ e $\delta \mathrm{R}_{\mathrm{TP}}$, apenas a primeira coluna da matriz $\Delta \mathbf{Z}-\widetilde{\mathbf{Z}}_{t} \widetilde{\mathbf{N}}_{t}^{-1} \Delta \mathbf{N}$ é utilizada em conseqüência da regra de Cramer (Hoffman \& Ray, 1971).

O coeficiente linearizado de uma onda P refletida considerando uma onda $\mathrm{P}$ incidente é então dado por:

$$
\begin{aligned}
& \mathrm{R}_{\mathrm{pP}}=8 \mathrm{~F}_{\mathrm{pp}}^{\mathrm{ISQ} Q}+\left\{\frac{\Delta\left(\mathrm{C}_{13}+2 \mathrm{C}_{55}-\mathrm{C}_{33}\right)}{\overline{\mathrm{C}}_{33}} \cos \varphi+\right. \\
& +\left[\frac{\Delta\left(\mathrm{C}_{23}+2 \mathrm{C}_{44}-\mathrm{C}_{33}\right)}{\overline{\mathrm{C}}_{33}}-8 \frac{\Delta\left(\mathrm{C}_{44}-\mathrm{C}_{5}\right)}{2 \overline{\mathrm{C}}_{33}}\right] \operatorname{sen}^{2} \varphi_{4}+ \\
& +2\left[\frac{\Delta\left(\mathrm{C}_{36}+2 \mathrm{C}_{45}\right)}{\overline{\mathrm{C}}_{33}}-4 \frac{\Delta \mathrm{C}_{45}}{\overline{\mathrm{C}}_{33}}\right] \cos \rho \operatorname{sen} \varphi \rho \operatorname{sen}^{2} \theta+ \\
& +\left\{\frac{\Delta\left(\mathrm{C}_{11}-\mathrm{C}_{33}\right)}{2 \overline{\mathrm{C}}_{33}} \cos \varphi+\frac{\Delta\left(\mathrm{C}_{22}-\mathrm{C}_{33}\right)}{2 \overline{\mathrm{C}}_{33}} \operatorname{set}^{4} \varphi+\right. \\
& +\frac{\Delta\left(\mathrm{C}_{12}+2 \mathrm{C}_{66}-\mathrm{C}_{33}\right)}{\overline{\mathrm{C}}_{33}} \cos ^{2} \varphi \operatorname{set}^{2} \varphi \\
& +2 \frac{\Delta \mathrm{C}_{16}}{\overline{\mathrm{C}}_{33}} \cos \varphi \operatorname{sen} \varphi+2 \frac{\Delta \mathrm{C}_{26}}{\overline{\mathrm{C}}_{33}} \cos \varphi \operatorname{ser}^{3} \varphi \rho \operatorname{ser}^{2} \operatorname{tatr}^{2} \theta
\end{aligned}
$$

em que $\delta \mathrm{R}_{\mathrm{PP}}^{(\mathrm{ISO})}$ representa a perturbação isotrópica do coeficiente $\delta \mathrm{R}_{\mathrm{PP}}$.

$\mathrm{O}$ coeficiente linearizado da onda $\mathrm{S}$ refletida considerando uma onda $P$ incidente é dado por:

$$
\begin{aligned}
& 8 \mathrm{R}_{\mathrm{Sp}}=8 \mathrm{~F}_{\mathrm{Sp}}^{\prime \mathrm{ISO}}+4\left(k \operatorname{sen}^{2} \theta-\mathrm{K}(\theta \cos \theta)\right. \\
& \left\{\frac{\Delta\left(\mathrm{C}_{44}-\mathrm{C}_{55}\right)}{2 \overline{\mathrm{C}}_{33}} \operatorname{sen}^{2} \varphi+\frac{\Delta \mathrm{C}_{45}}{\overline{\mathrm{C}}_{33}} \operatorname{sen} \varphi \cos \varphi\right\} \frac{\operatorname{sen} \theta}{\mathrm{K}(\theta)} \\
& +2\left\{\frac{\Delta\left(\mathrm{C}_{11}-\mathrm{C}_{33}\right)}{2 \overline{\mathrm{C}}_{33}} \cos \varphi+\frac{\Delta\left(\mathrm{C}_{22}-\mathrm{C}_{33}\right)}{2 \overline{\mathrm{C}}_{33}} \operatorname{sen}^{4} \varphi+\right. \\
& +\frac{\Delta\left(\mathrm{C}_{12}+2 \mathrm{C}_{66}-\mathrm{C}_{33}\right)}{\overline{\mathrm{C}}_{33}} \operatorname{sen}^{2} \varphi \cos ^{2} \varphi \\
& +2 \frac{\Delta \mathrm{C}_{16}}{\overline{\mathrm{C}}_{33}} \operatorname{sen} \varphi \cos \varphi+2 \frac{\Delta \mathrm{C}_{26}}{\overline{\mathrm{C}}_{33}} \operatorname{sen}^{3} \varphi \cos \varphi \frac{\operatorname{sen}^{3} \theta}{\eta(\theta)} \\
& +\left\{\frac{\Delta\left(\mathrm{C}_{13}+2 \mathrm{C}_{55}-\mathrm{C}_{33}\right)}{\overline{\mathrm{C}}_{33}} \cos \varphi+\right. \\
& +\frac{\Delta\left(\mathrm{C}_{23}+2 \mathrm{C}_{44}-\mathrm{C}_{33}\right)}{\overline{\mathrm{C}}_{33}} \operatorname{sen} \varphi
\end{aligned}
$$




$$
\begin{aligned}
& +2 \frac{\Delta\left(\mathrm{C}_{36}+2 \mathrm{C}_{45}\right)}{\overline{\mathrm{C}}_{33}} \operatorname{sen} \varphi \cos \varphi \frac{\operatorname{sen} \theta \cos 2 \theta}{\eta(\theta)} \\
& +\left\{\frac{\left.\Delta \mathrm{C}_{25}+2 \mathrm{C}_{46}\right)}{\overline{\mathrm{C}}_{33}} \operatorname{sen}^{2} \varphi \cos \varphi+\right. \\
& +\frac{\left.4 \mathrm{C}_{14}+2 \mathrm{C}_{56}\right)}{\overline{\mathrm{C}}_{33}} \operatorname{sen} \varphi \cos \varphi \\
& +\frac{\Delta \mathrm{C}_{15}}{\overline{\mathrm{C}}_{33}} \cos \varphi+\frac{\Delta \mathrm{C}_{24}}{\overline{\mathrm{C}}_{33}} \operatorname{sen}^{3} \varphi \frac{\operatorname{sen} \theta(2 \kappa \omega(\Theta)-1)}{\kappa \mathrm{K}(\Theta) \eta(\Theta)} \\
& +\left\{\frac{\Delta \mathrm{C}_{\mathrm{J}^{4}}}{\overline{\overline{\mathrm{C}}}_{\mathrm{J}}} \operatorname{sen} \varphi+\frac{\Delta \mathrm{C}_{\mathrm{j}}}{\overline{\mathrm{C}}_{\mathrm{J}}} \cos \varphi \frac{\cos \theta[\cos \theta-2 \mathrm{~K}(\theta) \omega(\theta)]}{\kappa \mathrm{K}(\theta) \eta(\theta)}\right. \text {; }
\end{aligned}
$$

em que $\delta \mathrm{R}_{\mathrm{SP}}^{(\mathrm{ISO})}$ representa a perturbação isotrópica do coeficiente $\delta \mathrm{R}_{\mathrm{SP}}$.

$\mathrm{O}$ coeficiente linearizado de uma onda $\mathrm{T}$ considerando uma onda $P$ incidente é dado por:

$$
\begin{aligned}
& \mathcal{E}_{\mathrm{TF}}=\frac{1}{\mathrm{kK}(\theta)}\left\{\left[\frac{\Delta\left(\mathrm{C}_{24}-2 \mathrm{C}_{56}\right)}{\overline{\mathrm{C}}_{33}} \cos \varphi \operatorname{sen}^{2} \varphi-\right.\right. \\
& \frac{-\Delta\left(\mathrm{C}_{15}-2 \mathrm{C}_{40}\right)}{\overline{\mathrm{C}}_{33}} \cos ^{2} \varphi \operatorname{sen} \varphi+ \\
& \left.\frac{\Delta C_{14}}{\overline{\mathrm{C}}_{33}} \cos \varphi-\frac{\Delta \mathrm{C}_{25}}{\overline{\mathrm{C}}_{33}} \operatorname{set}^{3} \varphi\right] \operatorname{set}^{2} \theta+ \\
& +\left[\frac{\Delta\left(\mathrm{C}_{44}-\mathrm{C}_{55}\right)}{\overline{\mathrm{C}}_{33}} \operatorname{sen} 2 \varphi+\frac{\Delta \mathrm{C}_{45}}{\overline{\mathrm{C}}_{33}} \cos 2 \varphi\right] \operatorname{sen} 2 \theta+ \\
& \left.\left[\frac{\Delta \mathrm{S}_{34}}{\overline{\mathrm{C}}_{33}} \cos \varphi-\frac{\Delta_{35}}{\overline{\mathrm{G}}_{3}} \operatorname{sen} \varphi\right] \cos ^{2} \theta\right\}- \\
& \frac{[\kappa \cos \theta-K(\theta)]}{K(\theta)\left(1-\kappa^{2}\right)}\left\{\left[\left(\frac{\left.4 C_{22}-C_{33}\right)}{2 \bar{C}_{33}} \operatorname{ser}^{2} \varphi-\right.\right.\right. \\
& \left.\frac{\left(\mathrm{C}_{11}-\mathrm{C}_{33}\right)}{2 \overline{\mathrm{C}}_{3}} \cos ^{2} \varphi\right) \operatorname{sen} 2 \varphi+ \\
& \frac{1}{2} \frac{4\left(\mathrm{C}_{12}+2 \mathrm{C}_{66}-\mathrm{C}_{33}\right)}{\overline{\mathrm{C}}_{3}} \operatorname{sen} 2 \varphi \cos 2 \varphi+ \\
& +\frac{\Delta \mathrm{C}_{16}}{\overline{\mathrm{C}}_{33}} \cos ^{2} \varphi\left(\cos ^{2} \varphi-3 \operatorname{sen}^{2} \varphi\right)+ \\
& \left.\frac{\Delta \mathrm{C}_{26}}{\overline{\mathrm{C}}_{33}} \operatorname{set}^{2} \varphi\left(3 \cos ^{2} \varphi-\operatorname{set}^{2} \varphi\right)\right] \operatorname{sen}^{3} \theta \\
& +\left[\frac{\Delta\left(\mathrm{G}_{4}+2 \mathrm{C}_{6}\right)}{\overline{\mathrm{C}}_{3}} \cos \varphi\left(\cos \varphi-2 \operatorname{set}^{2} \varphi\right)+\right. \\
& \frac{\Delta\left(\mathrm{C}_{2 s}+2 \mathrm{C}_{46}\right)}{\overline{\mathrm{C}}_{3}} \operatorname{ser}\left(2 \cos \varphi-\operatorname{set}^{2} p\right)+ \\
& \left.+\frac{3}{2}\left(\frac{\Delta \mathrm{C}_{24}}{\overline{\mathrm{C}}_{3}} \operatorname{ser} \varphi-\frac{\Delta \mathrm{C}_{15}}{\overline{\mathrm{G}}_{33}} \cos \varphi\right) \operatorname{sen} 2 \varphi\right] \cos \theta \operatorname{sen}^{2} \theta+
\end{aligned}
$$

$$
\begin{gathered}
{\left[\frac { 1 } { 2 } \left[\left(\frac{\Delta\left(\mathrm{C}_{23}+2 \mathrm{C}_{44}-\mathrm{C}_{33}\right)}{\overline{\mathrm{C}}_{33}}-\frac{\left.\Delta \mathrm{C}_{13}+2 \mathrm{C}_{55}-\mathrm{C}_{33}\right)}{\overline{\mathrm{C}}_{33}}\right) \operatorname{sen} 2 \varphi+\right.\right.} \\
\left.\frac{\left.\Delta \mathrm{C}_{36}+2 \mathrm{C}_{43}\right)}{\overline{\mathrm{C}}_{33}} \cos 2 \varphi\right] \cos \theta \operatorname{set} \theta+ \\
\left.\left[\frac{\Delta \mathrm{C}_{34}}{\overline{\mathrm{C}}_{33}} \cos \varphi-\frac{\Delta \mathrm{C}_{55}}{\overline{\mathrm{C}}_{33}} \operatorname{sen} \varphi\right] \cos \theta\right\}
\end{gathered}
$$

em que: $\varphi$ é o ângulo que determina o azimute, $\theta$ é o ângulo de incidência, $\kappa=\frac{\beta}{\alpha}$ é a razão entre as velocidades da onda longitudinal e cisalhantes do meio isotrópico de referência, $K(\theta)=\sqrt{1-\kappa^{2} \operatorname{sen}^{2} \theta}$ é o co-seno do ângulo de reflexão da onda convertida $S$,

$$
\begin{aligned}
& \omega(\theta)=\kappa \operatorname{sen}^{2} \theta+K(\theta) \cos \theta \\
& \eta(\theta)=K(\theta)+\kappa \cos \theta .
\end{aligned}
$$

As perturbações isotrópicas são dadas por:

$$
\begin{aligned}
\delta \mathrm{R}_{\mathrm{PP}}^{(\mathrm{ISO})}= & \frac{\Delta \mathrm{Z}}{\mathrm{Z}}+\frac{\Delta \alpha}{\alpha} \tan ^{2} \theta-4 \kappa^{2} \frac{\Delta \mathrm{G}}{\mathrm{G}} \operatorname{sen}^{2} \theta \\
\mathrm{e} & \quad \delta \mathrm{R}_{\mathrm{SP}}^{(\mathrm{SSO} 0)}=\frac{\operatorname{sen} \theta}{\mathrm{K}(\theta)}\left[\frac{\Delta \alpha}{\alpha}-\frac{\Delta \mathrm{Z}}{\mathrm{Z}}+\right. \\
& \left.2 \kappa\left(\operatorname{sen}^{2} \theta-\mathrm{K}(\theta) \cos \theta\right) \frac{\Delta \mathrm{G}}{\mathrm{G}}\right]
\end{aligned}
$$

em que : $Z=\rho \alpha$ é a impedância acústica do meio isotrópico de referência, $G=\rho \beta^{2}$ é o módulo de cisalhamento do meio de referência.

A perturbação isotrópica para a onda refletida $\mathrm{T}$ é nula uma vez que não há conversão de onda $\mathrm{P}$ para onda T no caso isotrópico. Para maiores detalhes sobre as fórmulas linearizadas ver apêndice $\mathrm{A}$.

\section{TESTES}

Nesta seção avaliamos as aproximações em um conjunto de modelos geologicamente plausíveis. A abordagem proposta apresenta limitações quando aplicados a problemas reais por causa das hipóteses simplificadas. Será feita uma análise de sensibilidade 
à violação das hipóteses de baixo contraste e fraca anisotropia. Os resultados obtidos foram satisfatórios.

As formas linearizadas dos coeficientes de reflexão $\mathrm{R}_{\mathrm{PP}}, \mathrm{R}_{\mathrm{SP}}$ e $\mathrm{R}_{\mathrm{TP}}$ foram avaliadas da seguinte forma. Primeiro, os coeficientes obtidos através das equações de Zoeppritz e as aproximações lineares foram representados em estereogramas. A seguir é mostrado o estereograma do erro. Para o caso dos coeficientes $\mathrm{R}_{\mathrm{PP}}$ e $\mathrm{R}_{\mathrm{SP}}$ determinou-se o erro percentual relativo em geral, em alguns casos optouse por determinar o erro absoluto de $\mathrm{R}_{\mathrm{SP}}$ e para o coeficiente $\mathrm{R}_{\mathrm{TP}}$ determinou-se erro absoluto. A determinação do erro absoluto ao invés do erro relativo deve-se ao fato de que no caso da onda $\mathrm{T}$, nos planos de simetria não há conversão de onda $\mathrm{P}$ em $\mathrm{T}$, o que torna a fórmula do erro relativo para este último coeficiente inválida e para alguns casos da onda S o coeficiente de $\mathrm{R}_{\mathrm{SP}}$ assume grandes valores quando comparados com os outros valores assumidos por este coeficiente invalidando o erro relativo nestes casos. Nos estereogramas, os coeficientes foram calculados para ângulos de incidência de até $40^{\circ}$, a partir da normal à interface plana que separa os meios e para uma variação azimutal de $360^{\circ}$.

O modelo I caracteriza-se pela fraca anisotropia e baixos contrastes entre os parâmetros elásticos dos meios envolvidos e corresponde ao modelo A-C apresentado em Vavrycuk \& Psencik(1998). O meio incidente é isotrópico com densidade $\rho_{\mathrm{I}}=2,65 \mathrm{~g} / \mathrm{cm}^{3}$ e as velocidades das ondas P e S são dadas por $\alpha_{I}=4,00 \mathrm{~km} / \mathrm{s}, \beta_{\mathrm{I}}=2,31 \mathrm{~km} / \mathrm{s}$, respectivamente. O meio subjacente é um TIH (meio transversalmente isotrópico com eixo de simetria horizontal (ver Musgrave, 1970) e corresponde ao meio efetivo associado a um sistema de inclusões elipsoidais paralelas em uma formação isotrópica, segundo Hudson (1982). O meio isotrópico de referência tem densidade $\rho_{\text {ref }}=2,60 \mathrm{~g} / \mathrm{cm}^{3}$ e as velocidades das ondas $\mathrm{P}$ e $\mathrm{S}$ são dadas por $\alpha_{\text {ref }}=4,00 \mathrm{~km} / \mathrm{s} \quad$ e $\quad \beta_{\text {ref }}=2,31 \mathrm{~km} / \mathrm{s}$, respectivamente. A razão de aspecto dos elipsóides é de $10^{-4} \mathrm{e}$ a densidade de fraturamento de 0,05 . Este meio é caracterizado pelo tensor elástico:

$$
\mathrm{C}_{o \&}=\left[\begin{array}{cccccc}
31,10 & 10,37 & 10,37 & 0,00 & 0,00 & 0,00 \\
& 40,43 & 12,69 & 0,00 & 0,00 & 0,00 \\
& & 40,43 & 0,00 & 0,00 & 0,00 \\
& & & 13,86 & 0,00 & 0,00 \\
& & & & 12,38 & 0,00 \\
& & & & & 12,38
\end{array}\right]
$$

Neste modelo verifica-se que as fórmulas linearizadas dos coeficientes de reflexão $R_{P P}, R_{S P} e$ $\mathrm{R}_{\mathrm{TP}}$ apresentam-se muito próximas da formulação exata para ângulos de incidência de até $30^{\circ}$, uma vez que os erros relativos dos coeficientes linearizados $\mathrm{R}_{\mathrm{PP}}$ e $\mathrm{R}_{\mathrm{SP}}$ são inferiores à $6 \%$ e 10\% respectivamente enquanto que a forma linearizada $\mathrm{R}_{\mathrm{TP}}$ apresenta erro absoluto da ordem de 0,001 . Isto já era esperado uma vez que este modelo obedece aos pressupostos considerados na derivação destas linearizações.

O modelo II caracteriza-se pela forte anisotropia e fraco contraste dos parâmetros elásticos. O meio incidente é isotrópico com densidade de $\rho_{\mathrm{I}}=2,20 \mathrm{~g} / \mathrm{cm}^{3}$ e as velocidades das ondas P e S dadas por $\alpha_{\mathrm{I}}=4,23 \mathrm{~km} / \mathrm{s}, \quad \beta_{\mathrm{I}}=2,73 \mathrm{~km} / \mathrm{s}$ respectivamente. O meio subjacente é um TIH que corresponde às propriedades de um folhelho com forte anisotropia (Thomsen, 1986), e que foi girado de $30^{\circ}$ em relação ao eixo x e $60^{\circ}$ em relação ao eixo $\mathrm{z}$ e tem como tensor elástico:

$$
C_{凶 \psi}=\left[\begin{array}{cccccc}
66,66 & 39,42 & 19,62 & 0,00 & 0,00 & 0,00 \\
& 39,96 & 39,49 & 0,00 & 0,00 & 0,00 \\
& & 66,66 & 0,00 & 0,00 & 0,00 \\
& & & 10,94 & 0,00 & 0,00 \\
& & & & 23,52 & 0,00 \\
& & & & & 10,94
\end{array}\right]
$$

A formulação exata de $\mathrm{R}_{\mathrm{PP}}$ é dada pela figura 1. A formulação linearizada (Fig. 2) apresenta-se muito próxima da formulação exata para incidências de até $30^{\circ}$, já que o erro relativo para essas incidências é inferior a $7 \%$ (Fig. 3). Verifica-se que o coeficiente $\mathrm{R}_{\mathrm{PP}}$ exato e linearizado não atestam o mergulho. Isto mostra que em modelos com mergulho este coeficiente não possui informações sobre o mesmo. Esse tipo de informação é obtido através das ondas convertidas. Na figura 4 tem-se a formulação exata do coeficiente $R_{S P}$ e verifica-se que a formulação linearizada (Fig. 5) apresenta-se muito próxima da 




Figura 1 - Estereograma com o coeficiente de reflexão $R_{P P}$ exato. $O$ azimute varia de $0^{0}$ até $360^{\circ}$ e está representado no contorno da figura. A incidência varia de $0^{\circ}$ até $40^{\circ}$ a partir da normal à interface que separa os planos, representada na figura pelos números internos ao diagrama. A escala de cinza mostra o valor absoluto do coeficiente. Verifica-se pela figura que este coeficiente tem simetria ortorrômbica o que mostra que o coeficiente é insensível ao mergulho.

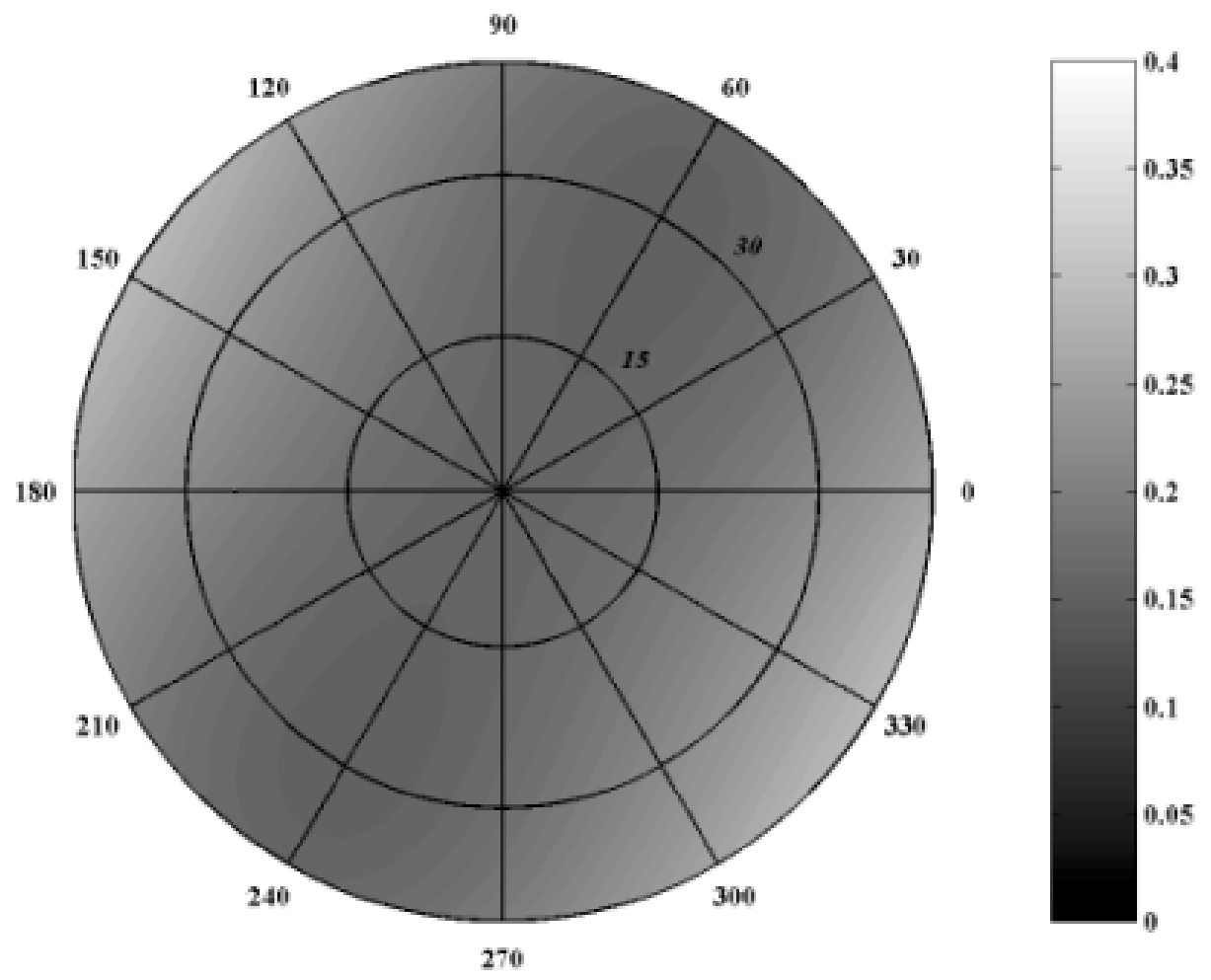

Figura 2 - Aproximação linear do coeficiente de reflexão $\mathrm{R}_{\mathrm{PP}}$. Como na formulação exata, a forma linearizada é insensível ao mergulho. 


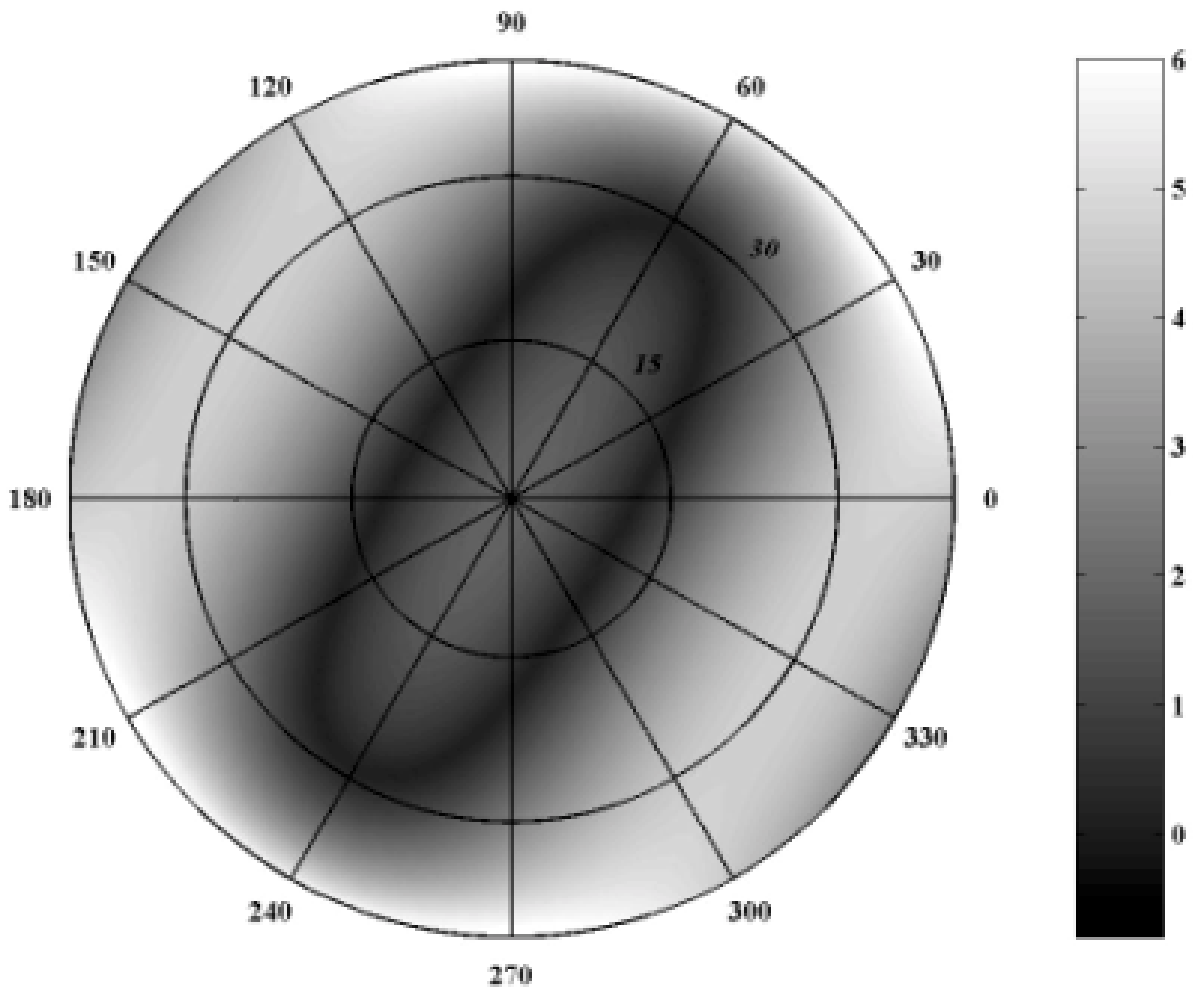

Figura 3 - Erro percentual relativo da aproximação linear de $\mathrm{R}_{\mathrm{PP}}$. De acordo com o erro verifica-se bom ajuste da forma linearizada para incidências de até $30^{\circ}$.

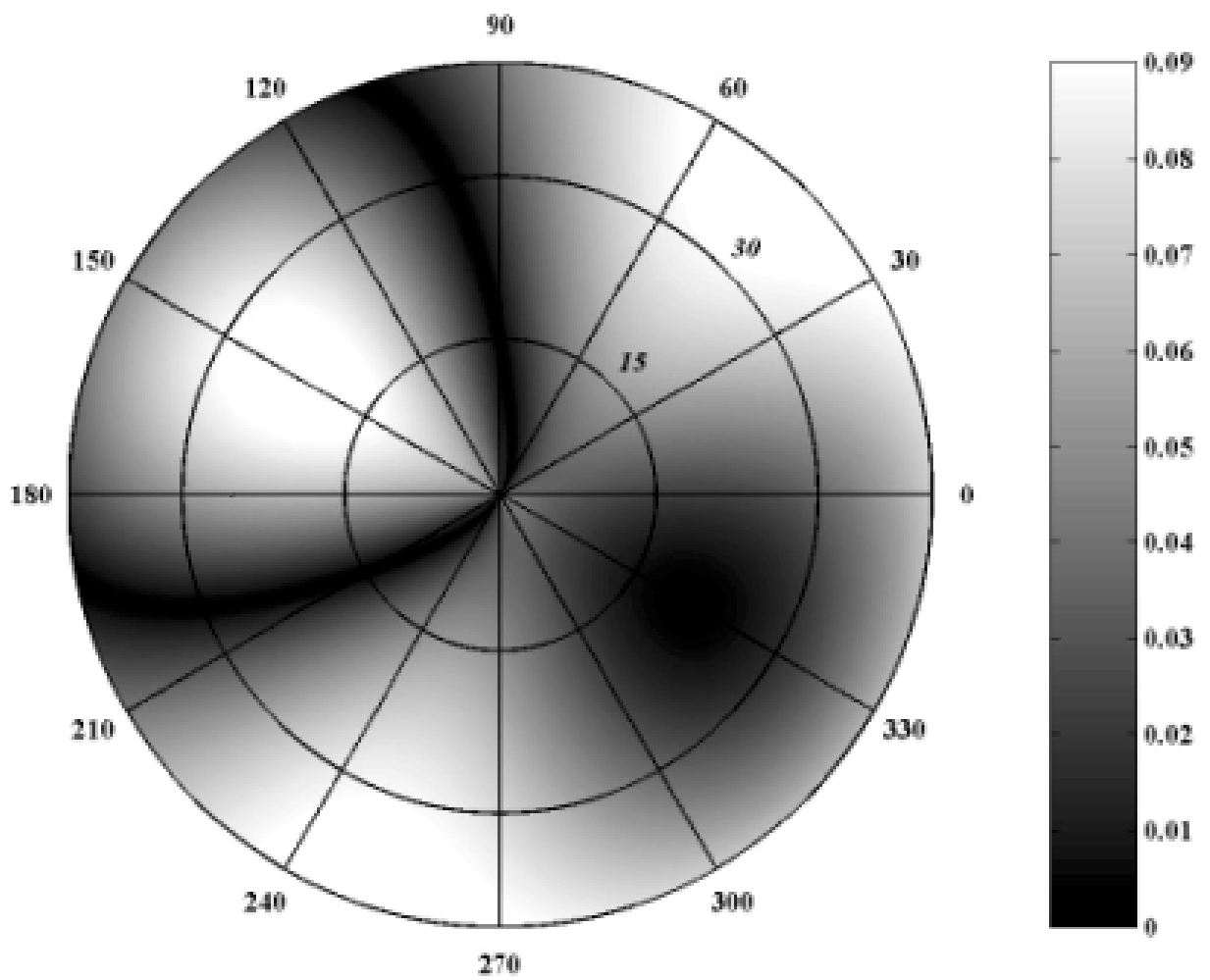

Figura 4 - Coeficiente de reflexão $\mathrm{RR}_{\mathrm{SP}}$ exato. Este coeficiente mostra-se sensível ao mergulho. 


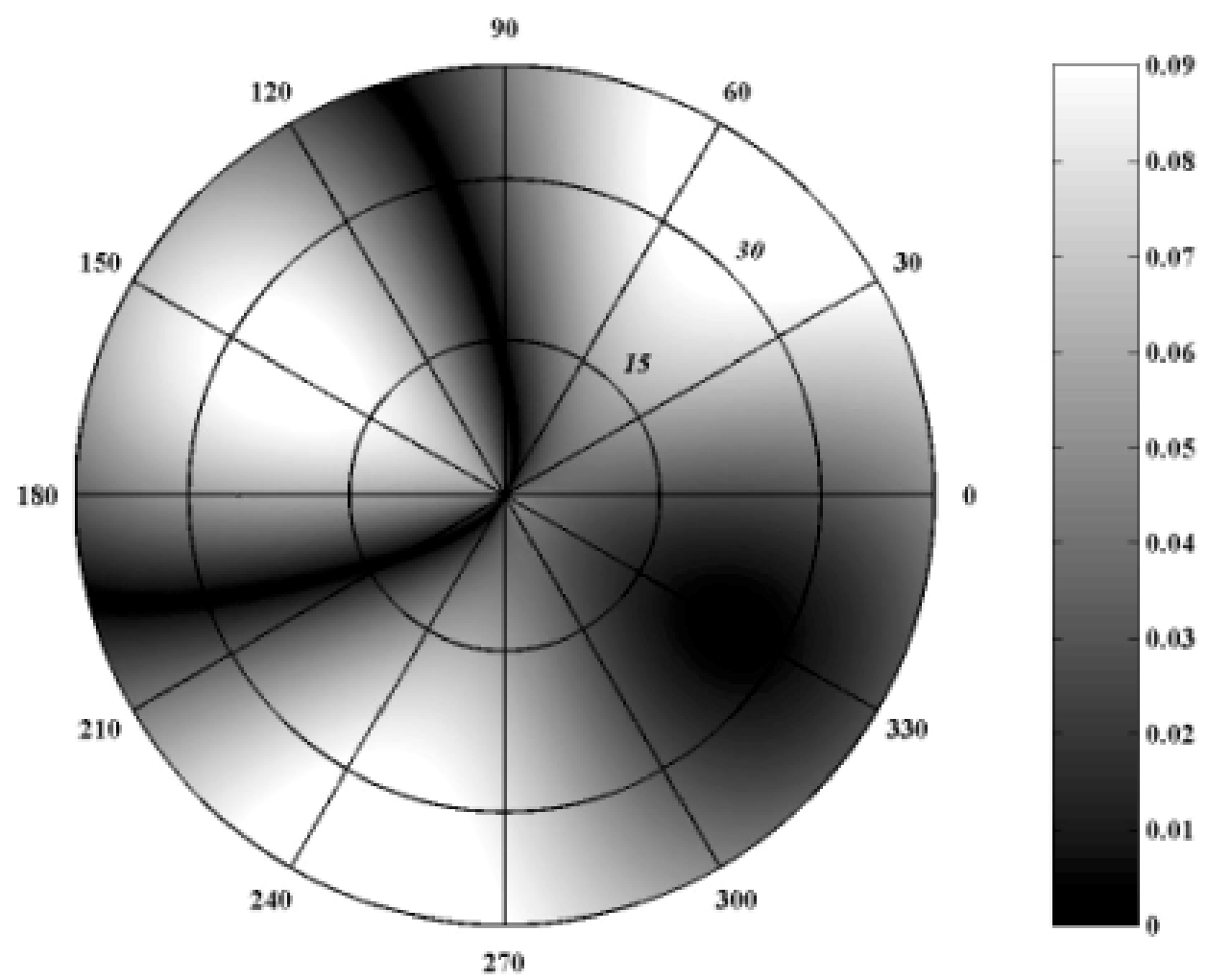

Figura 5 - Aproximação linear do coeficiente de reflexão $\mathrm{R}_{\mathrm{SP}}$. Formulação mostra-se sensível ao mergulho mostrando a consistência da linearização.

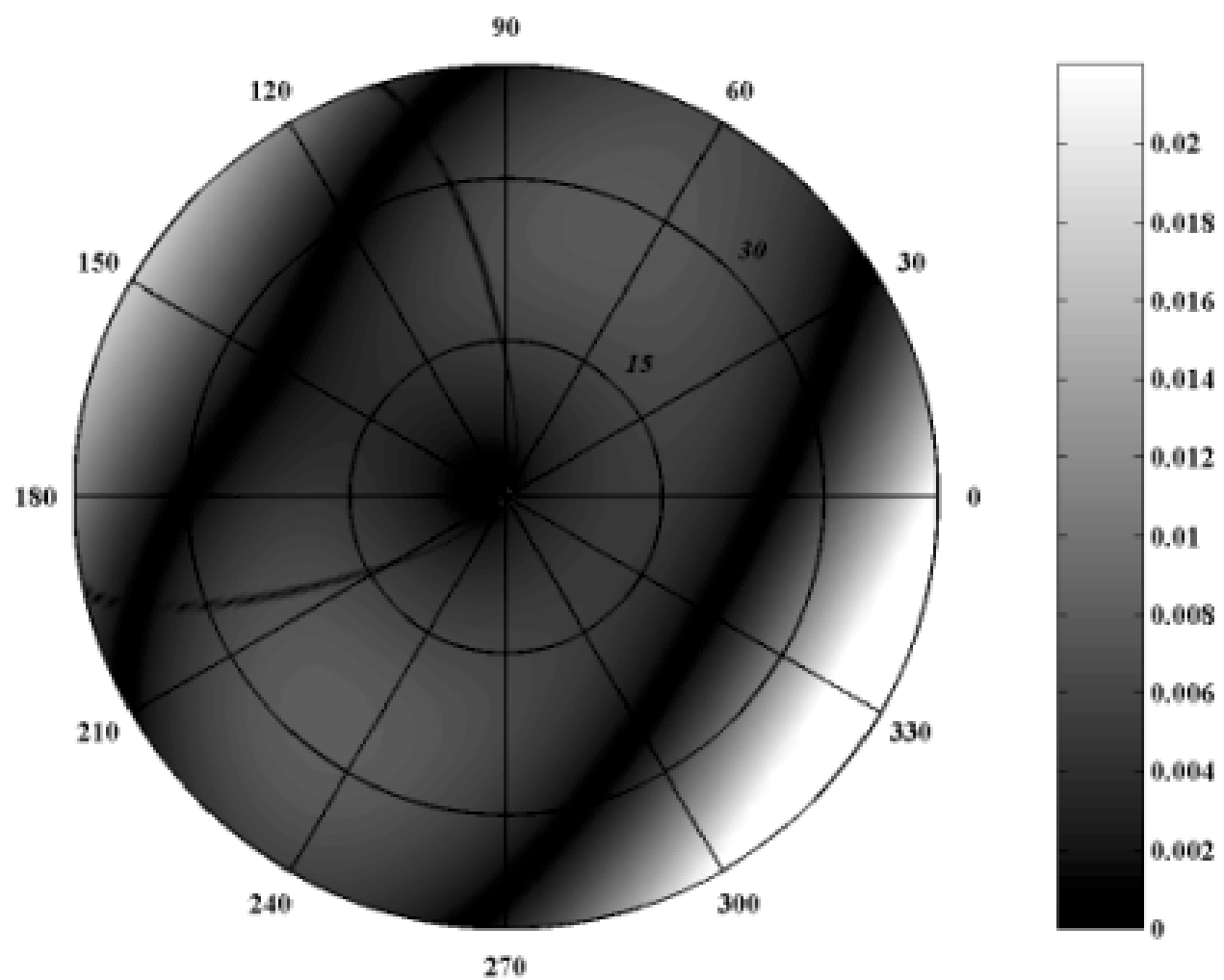

Figura 6 - Erro absoluto da aproximação linear $\mathrm{R}_{\mathrm{SP}}$. A escala foi mudada em relação as figuras 4 e 5 para melhor visualização do erro. Temse um bom ajuste (erro inferior a 0,005 ) da forma linearizada com relação à formulação exata para incidências de até $15^{\circ}$. 




Figura 7 - Coeficiente de reflexão $\mathrm{R}_{\mathrm{TP}}$ exato. Não há conversão de onda T no único plano de simetria do meio com mergulho.

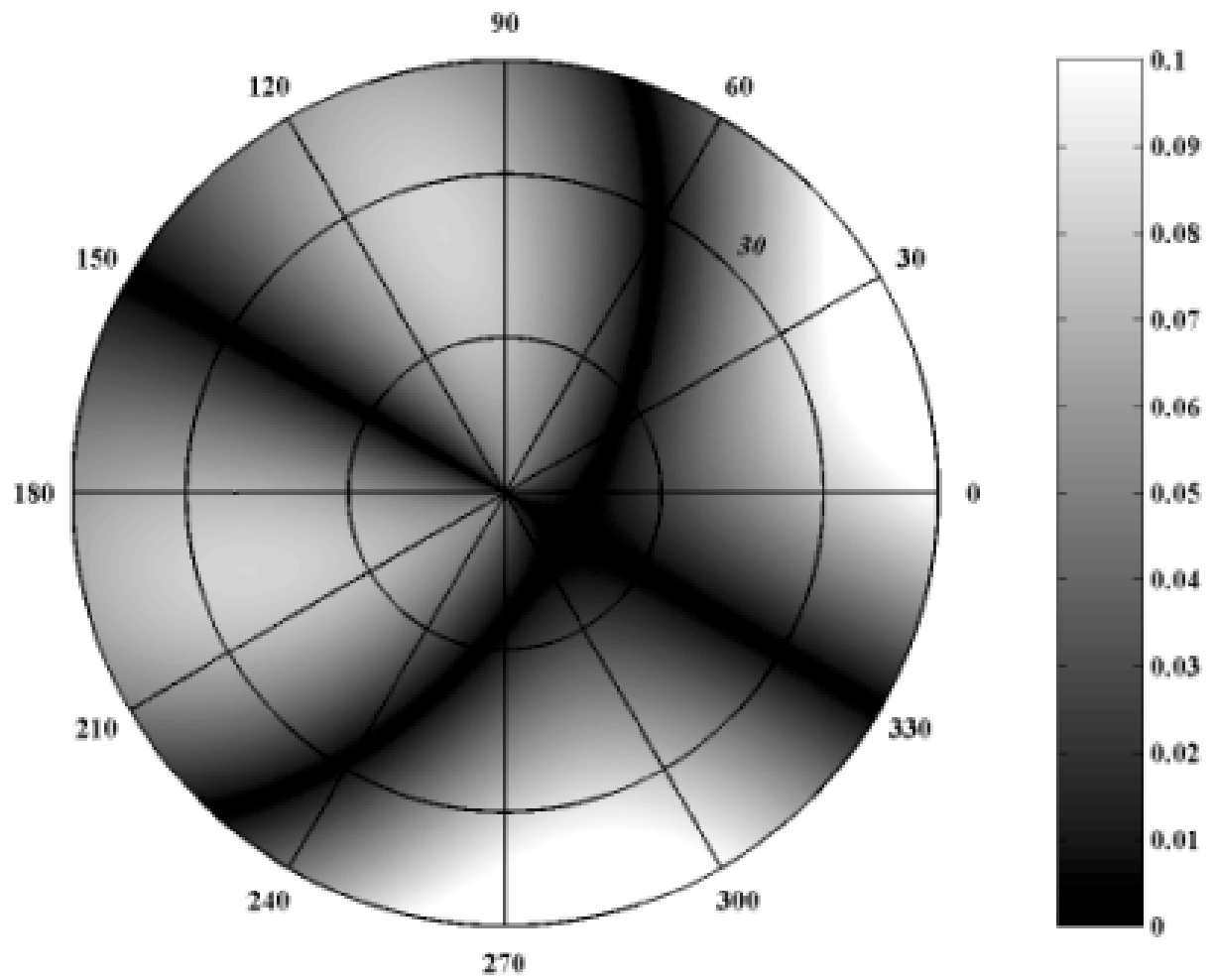

Figura 8 - Aproximação linear do coeficiente $\mathrm{R}_{\mathrm{TP}}$. A forma linearizada mostra que não há conversão de onda $\mathrm{P}$ em onda $\mathrm{T}$ no único plano de simetria o que mostra a consistência da formulação linear. 


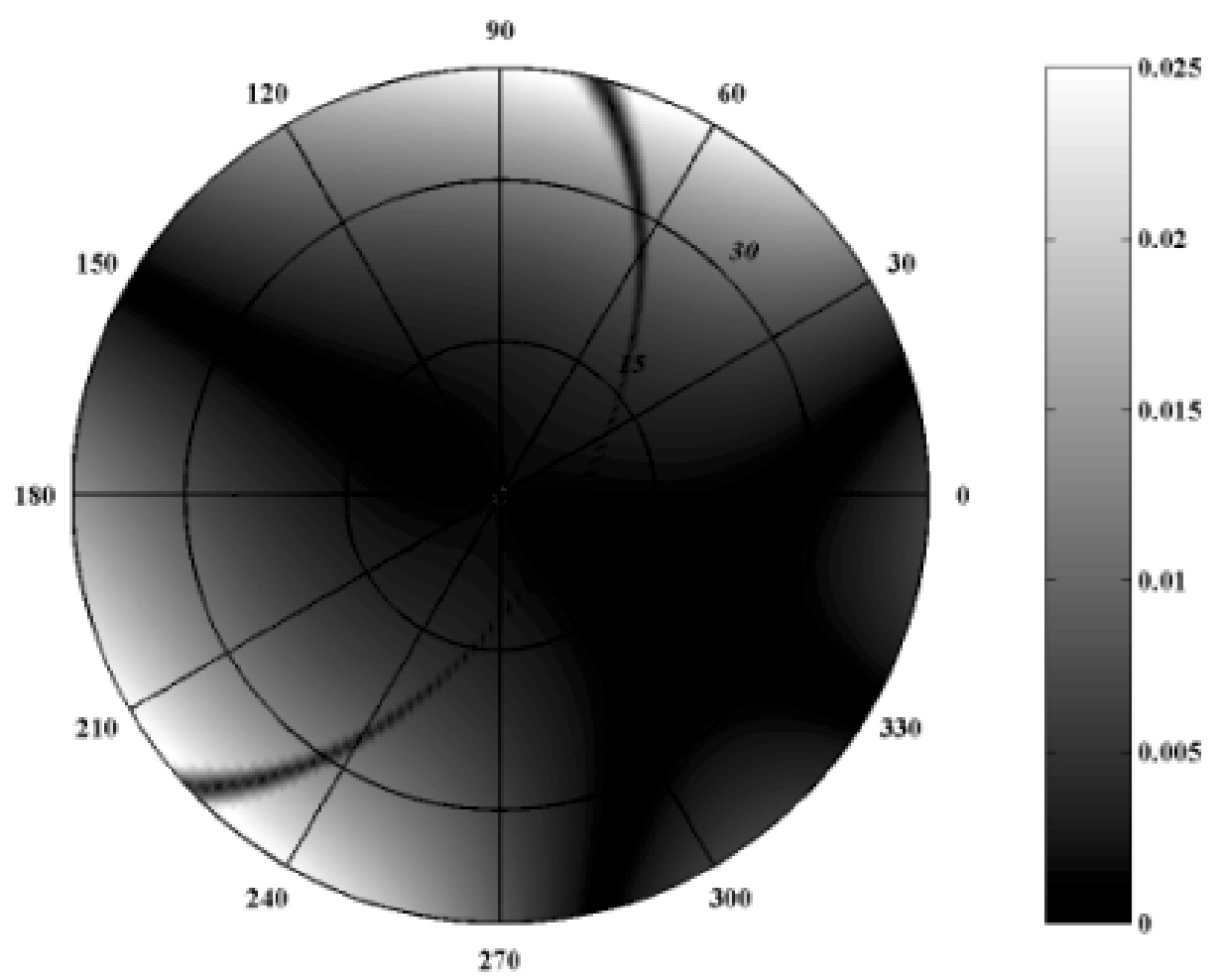

Figura 9 - Erro absoluto da aproximação linear $\mathrm{R}_{\mathrm{TP}}$. A escala foi mudada em relação as figuras 7 e 8 para melhor visualização do erro. Os erros nas regiões com incidência de até $30^{\circ}$ são inferiores a 0,02 . O ângulo de incidência está variando de $0^{\circ}$ até $40^{\circ}$ a partir da normal à interface que separa os planos, representada na figura pelos arcos internos ao diagrama.

exata com erros absolutos em torno a 0,005 para incidências de até $15^{\circ}$ (Fig. 6). Para o caso do coeficiente $\mathrm{R}_{\mathrm{TP}}$ verifica-se pela formulação exata (Fig. 7) que não há conversão de onda T no plano de simetria e em regiões próximas a esse plano. A formulação linearizada apresenta bons resultados para incidências de até $30^{\circ}$ (Fig. 8). O erro absoluto para essas incidências é menor que 0,02 (Fig. 9). Conclui-se que pelo menos para este modelo a forte anisotropia não compromete as aproximações.

O modelo III caracteriza-se por forte contraste e fraca anisotropia. O meio incidente é isotrópico com densidade de $\rho_{\mathrm{I}}=2,0 \mathrm{~g} / \mathrm{cm}^{3}$ e velocidades das ondas $\mathrm{P}$ e $\mathrm{S}$ são de $\alpha_{\mathrm{I}}=2,5 \mathrm{~km} / \mathrm{s} \quad$ e $\beta_{\mathrm{I}}=1,20 \mathrm{~km} / \mathrm{s}$, respectivamente. O meio subjacente tem densidade de $\rho=2,6 \mathrm{~g} / \mathrm{cm}^{3}$ e o tensor elástico é dado por (16). O erro relativo da forma linearizada de $\mathrm{R}_{\mathrm{PP}}$ só é aceitável para incidências subnormais (incidências menores que $15^{\circ}$ ) e nestas incidências o erro é menor que 3\%. Para incidências maiores o erro é superior a $10 \%$. Na forma linearizadas $R_{S P}$ verifica-se que para incidências maiores que as subnormais o erro relativo é superior a $15 \%$ e para a forma linearizada e $\mathrm{R}_{\mathrm{TP}}$ para incidências de até $15^{\circ} \mathrm{O}$ erro absoluto é menor que 0,02 . Conclui-se que para este modelo as formas linearizadas só são razoáveis para incidências subnormais.

O modelo IV caracteriza-se por apresentar forte contraste e forte anisotropia. $\mathrm{O}$ meio incidente é um isotrópico cuja densidades é $\rho_{\mathrm{I}}=1,80 \mathrm{~g} / \mathrm{cm}^{3}$ e as velocidades das ondas P e S são $\alpha_{\mathrm{I}}=2,00 \mathrm{~km} / \mathrm{s}$ e $\beta_{\mathrm{I}}=1,20 \mathrm{~km} / \mathrm{s}$ respectivamente. O meio subjacente é um TIH com densidade $\rho=2,59 \mathrm{~g} / \mathrm{cm}^{3}$ e o tensor elástico é dado por (17). Da mesma forma que o modelo anterior às aproximações linearizadas só apresentam bom ajuste para incidências subnormais.

Foram feitos testes considerando o meio incidente anisotrópico. Quando o meio de incidência tem simetria azimutal os resultados não diferiram dos modelos que foram apresentados, isto é, para modelos que obedecem aos pressupostos de fraco contraste e fraca anisotropia os resultados das linearizações ajustam-se de forma satisfatória à formulação exata apresentando erros aceitáveis em 
relação ao nível de ruído associado à atual tecnologia de estimativa destes coeficientes. Quando o meio de incidência não apresenta simetria azimutal as aproximações não são satisfatórias pois o pressuposto da direção de polarização das ondas S e T em SV e SH respectivamente, não é válido. Para anisotropia e contrastes moderados as linearizações ainda apresentam-se satisfatórias para incidências de até $30^{\circ}$, considerando forte contraste e forte anisotropia as aproximações são aceitáveis apenas para incidência subnormal.

\section{CONSIDERAÇÕES FINAIS}

As aproximações lineares para $\mathrm{R}_{\mathrm{PP}}, \mathrm{R}_{\mathrm{SP}}$ e $\mathrm{R}_{\mathrm{TP}}$ apresentam um bom desempenho quando os modelos obedecem aos pressupostos utilizados nas linearização, ou seja, baixos contrastes e fraca anisotropia. Mesmo para modelos que violam uma destas condições as aproximações ainda são válidas desde que as incidências sejam próximas da normal à interface. Nos testes realizados verificou-se que:

$\mathrm{Na}$ violação dos pressupostos utilizados na obtenção das linearizações as aproximações apresentam melhor desempenho quando a hipótese de fraca anisotropia é violada do que quando a hipótese de fraco contraste é violada.

$O$ coeficiente $R_{P P}$ mostrou-se insensível ao mergulho das fraturas.

Em todos os exemplos excluiu-se as regiões muito próximas a ângulos críticos onde o comportamento da refletividade é altamente não linear como já havia sido observado em modelos isotrópicos por Castagna (1992).

\section{CONCLUSÕES}

Neste trabalho foi apresentada uma metodologia para a determinação da fórmula exata dos coeficientes de reflexão e transmissão para meios anisotrópicos arbitrários através das matrizes de impedância e polarização. A partir desta formulação obteve-se uma aproximação para os coeficientes de reflexão de uma onda $\mathrm{P}$ incidente considerando-se contraste fraco entre as propriedades físicas do meio e fraca anisotropia.
A metodologia apresentada para a determinação da formula exata dos coeficientes de reflexão e transmissão possui vantagens computacionais uma vez que todas as características do espalhamento podem ser obtidas de operações com matrizes de terceira ordem. A representação dos coeficientes de espalhamento utilizando de matrizes de polarização e impedância tem vantagens analíticas pois a partir desta formulação pôde-se determinar explicitamente as matrizes de reflexão e transmissão.

Nas linearizações do coeficiente $R_{P P}$ verificouse que algumas informações sobre os meios não podem ser recuperadas, enquanto que, as linearizações dos coeficientes $R_{S P}$ e $R_{T P}$ possuem informações sobre todos os parâmetros elásticos do meio. Assim as formas linearizadas são importantes na caracterização de ambigüidades para o problema de inversão destes coeficientes de reflexão. Além disso, estas linearizações possuem a vantagem de permitir uma análise mais simples de como os parâmetros ou combinação de parâmetros que caracterizam os meios influenciam nestes coeficientes.

A metodologia proposta pode ser aplicada mesmo em casos de contraste moderado de impedância e anisotropia dentro dos limites esperados em rochas sedimentares. Verificou-se ainda que o maior contraste entre os meios deteriora mais as aproximações que a presença de maior anisotropia.

As aproximações apresentadas neste trabalho, foram obtidas a partir dos pressupostos de fraco contraste entre as propriedades elásticas dos meios e anisotropia fraca. Entretanto os limites de validade destas fórmulas não podem ser caracterizados a partir de sua dedução. Uma analise que estabeleça esses limites é necessária para se determinar a sua utilização na interpretação de AVO/AVD.

A partir destas fórmulas linearizadas pode-se estimar as propriedades de meios na subsuperfície como por exemplo à orientação do plano de fratura e assim caracterizar a direção de maior permeabilidade.

\section{APÊNDICE A}

A linearização dos coeficientes de reflexão de uma onda incidente $\mathrm{P}$ foi feita no plano de azimute 
zero $(\varphi=0)$ e posteriormente estendidas para azimutes arbitrários através de rotação em torno do eixo vertical $\mathrm{x}_{3}$. Para tornar mais claro as fórmulas apresentadas nesta seção optamos por indicar o tipo de onda na forma de sobrescrito.

Considerando a equação (8) é necessário determinar a matriz $\left(\widetilde{\mathbf{Z}}_{r}-\widetilde{\mathbf{Z}}_{t} \widetilde{\mathbf{N}}_{t}^{-1} \widetilde{\mathbf{N}}_{r}\right)$ no meio isotrópico de referência e a primeira coluna da matriz de perturbação $\left(\Delta \mathbf{Z}-\widetilde{\mathbf{Z}}_{t} \widetilde{\mathbf{N}}_{t}^{-1} \Delta \mathbf{N}\right)$. A avaliação desta última expressão requer a determinação das perturbações da polarização da onda $\mathrm{P}$ incidente, $\delta \mathbf{n}^{P}$, e da perturbação da terceira componente da vagarosidade da onda $\mathrm{P}$ incidente, $\delta \mathrm{s}_{3}^{\mathrm{P}}$, em cada um dos meios.

\section{Determinação da matriz $\left(\widetilde{\mathbf{Z}}_{r}-\widetilde{\mathbf{Z}}_{t} \widetilde{\mathbf{N}}_{t}^{-1} \widetilde{\mathbf{N}}_{r}\right)$}

As velocidades e a densidade do meio isotrópico de referência são dadas por (7) e $\widetilde{\mathrm{N}}_{\mathrm{m} \alpha}=\widetilde{\mathrm{n}}_{\mathrm{m} \alpha}$, $\widetilde{Z}_{\mathrm{m} \alpha}=\widetilde{\mathrm{c}}_{3 \mathrm{mjl}} \widetilde{\mathrm{s}}_{\mathrm{l} \alpha} \widetilde{\mathrm{n}}_{\mathrm{j} \alpha}$ são as matrizes de polarização e impedância para o meio isotrópico de referência.

A matriz de polarização associada a transmissão no meio de referência isotrópico foi escolhida na forma:

$$
\widetilde{\mathbf{N}}_{t}=\left[\begin{array}{ccc}
\operatorname{sen} \theta & 0 & K(\theta) \\
0 & -1 & 0 \\
\cos \theta & 0 & -\kappa \operatorname{sen} \theta
\end{array}\right],
$$

em que escolhemos para polarização da onda $\mathrm{T}$ no meio de referência a direção perpendicular ao plano sagital (Helbig, 1994), onda SH, e a polarização da onda S é perpendicular a direção de incidência da onda P no plano sagital, onda SV. Esta escolha é arbitrária e pode não ser a mais conveniente quando o meio de incidência é anisotrópico e não apresenta simetria azimutal. Como o meio de referência é isotrópico e portanto possui plano de simetria especular, a matriz de polarização das ondas refletidas neste meio é obtida através da reflexão de (18) em relação a interface.
Com estas escolhas obtêm-se:

$$
\begin{aligned}
\left(\widetilde{\mathrm{Z}}_{r}-\widetilde{\mathrm{Z}}_{t} \widetilde{\mathrm{N}}_{t}^{-1} \widetilde{\mathrm{N}}_{r}\right]^{-1}=\frac{1}{2 \rho \kappa K(\theta) \cos \theta} \\
\quad\left[\begin{array}{ccc}
-\kappa K(\theta) \sin \theta & 0 & \kappa K(\theta) \cos \theta \\
0 & \cos \theta & 0 \\
-K(\theta) \cos \theta & 0 & \kappa \sin \theta \cos \theta
\end{array}\right]
\end{aligned}
$$

Determinação perturbações $\delta n^{P}$ e $\delta \mathrm{s}_{3}^{\mathrm{P}}$.

As perturbações da direção de polarização $\delta \mathbf{n}^{P}$ e da componente vertical da vagarosidade da onda $P$ incidente $\delta \mathrm{s}_{3}^{\mathrm{P}}$ em cada meio são obtidas a partir da equação de Christoffel,

$$
\Gamma_{\mathrm{kl}} \mathrm{n}_{1}^{\mathrm{P}}=\rho \mathrm{n}_{\mathrm{k}}^{\mathrm{P}} .
$$

A perturbação de primeira ordem da matriz de Chistoffel é dada por:

$$
\Gamma_{\mathrm{kl}} \delta \mathrm{n}_{1}^{\mathrm{P}}+\delta \Gamma_{\mathrm{kl}} \mathrm{n}_{1}^{\mathrm{P}}=\rho \delta \mathrm{n}_{\mathrm{k}}^{\mathrm{p}}+\delta \rho \mathrm{n}_{\mathrm{k}}^{\mathrm{P}},
$$

em que $\delta \Gamma_{\mathrm{kl}}$ é a matriz de Christoffel perturbada e $\delta \rho$ é a perturbação da densidade.

A perturbação $\delta s_{3}^{P}$ é obtida efetuando o produto interno de (21) com o vetor de polarização $\mathrm{n}_{\mathrm{k}}^{\mathrm{P}}$ o que resulta na relação:

$$
\mathrm{n}_{\mathrm{k}}^{\mathrm{P}} \delta \Gamma_{\mathrm{kj}} \mathrm{n}_{\mathrm{j}}^{\mathrm{P}}=\delta \rho .
$$

A perturbação $\delta \Gamma_{\mathrm{kl}}$ é dada pela expressão:

$\delta \Gamma_{\mathrm{kl}}=\delta \mathrm{c}_{\mathrm{kmlj}} \mathrm{s}_{\mathrm{m}} \mathrm{s}_{\mathrm{j}}+\mathrm{c}_{\mathrm{kmlj}} \delta \mathrm{s}_{\mathrm{m}} \mathrm{s}_{\mathrm{j}}+\mathrm{c}_{\mathrm{kmlj}} \mathrm{s}_{\mathrm{m}} \delta \mathrm{s}_{\mathrm{j}}$,

em que $\delta c_{\text {kmlj }}$ são as perturbações do meio em relação ao meio de referência. Utilizando (22) e (23) podemos obter imediatamente $\delta \mathrm{s}_{3}^{\mathrm{P}}$.

O cálculo de $\delta \mathbf{n}^{P}$ é obtido através do sistema formado pelo produto interno de (21) com os vetores de polarização $\mathrm{n}_{\mathrm{k}}^{\mathrm{S}}$ e $\mathrm{n}_{\mathrm{k}}^{\mathrm{T}}$ respectivamente e da condição de normalização $\mathbf{n}^{P}$, conforme mostrado abaixo : 


$$
\left\{\begin{array}{c}
\mathrm{n}_{\mathrm{k}}^{\mathrm{S}}\left(\Gamma_{\mathrm{kl}}-\rho \delta_{\mathrm{kl}}\right) \delta \mathrm{n}_{1}^{\mathrm{P}}=\mathrm{n}_{\mathrm{k}}^{\mathrm{S}} \delta \Gamma_{\mathrm{kl}} \mathrm{n}_{1}^{\mathrm{P}}+\mathrm{n}_{\mathrm{k}}^{\mathrm{S}} \delta \rho \mathrm{n}_{1}^{\mathrm{P}} \\
\mathrm{n}_{\mathrm{k}}^{\mathrm{T}}\left(\Gamma_{\mathrm{k} l}-\rho \delta_{\mathrm{kl}}\right) \delta \mathrm{n}_{1}^{\mathrm{P}}=\mathrm{n}_{\mathrm{k}}^{\mathrm{T}} \delta \Gamma_{\mathrm{kl}} \mathrm{n}_{1}^{\mathrm{P}}+\mathrm{n}_{\mathrm{k}}^{\mathrm{T}} \delta \rho \mathrm{n}_{1}^{\mathrm{P}} \\
\mathrm{n}_{\mathrm{k}}^{\mathrm{P}} \delta \mathrm{n}_{\mathrm{k}}^{\mathrm{P}}=0
\end{array}\right.
$$

em que $\delta_{\mathrm{ij}}$ é o delta de Kronecker. Da solução do sistema linear acima tem-se a perturbação da polarização para uma onda incidente $P$.

A seguir, conhecidos $\delta \mathrm{s}_{3}^{\mathrm{P}}$ e $\delta \mathbf{n}^{P}$ a primeira coluna da matriz de impedância perturbada é avaliada em cada meio através da expressão:

$$
\begin{aligned}
\delta Z_{\mathrm{kp}}= & \frac{\delta \mathrm{C}_{3 \mathrm{kjl}}}{\alpha} \mathrm{n}_{1}^{\mathrm{p}} \mathrm{n}_{\mathrm{j}}^{\mathrm{p}}+(\lambda+\mu) \delta \mathrm{s}_{3}^{\mathrm{p}} \mathrm{n}_{3}^{\mathrm{p}} \delta_{\mathrm{k} 3}+ \\
& +\frac{\lambda}{\alpha}\left[\mathrm{n}_{\mathrm{j}}^{\mathrm{p}} \delta \mathrm{n}_{\mathrm{j}}^{\mathrm{p}}+\mathrm{n}_{3}^{\mathrm{p}} \delta \mathrm{n}_{3}^{\mathrm{p}}\right) \delta_{\mathrm{k} 3} \\
+ & \frac{\mu}{\alpha}\left[\mathrm{n}_{\mathrm{j}}^{\mathrm{p}} \delta \mathrm{n}_{\mathrm{j}}^{\mathrm{p}}+\mathrm{n}_{3}^{\mathrm{p}} \delta \mathrm{n}_{3}^{\mathrm{p}}\right)+\mu \delta \delta_{3}^{\mathrm{p}} \mathrm{n}_{\mathrm{k}}^{\mathrm{p}}
\end{aligned}
$$

em que: $\lambda$ e $\mu$ são os parâmetros de Lamé.

Finalmente os coeficientes linearizados $\delta \mathrm{R}_{\mathrm{PP}}, \delta \mathrm{R}_{\mathrm{SP}}$ e $\delta R_{T P}$ são então obtidos da primeira equação do sistema (8).

No caso de um azimute arbitrário $\varphi$ em relação ao eixo $\mathrm{x}_{1}$, os parâmetros elásticos são obtidos a partir da rotação em torno do eixo vertical $\mathrm{x}_{3}$ (Helbig, 1994). E os coeficientes de reflexão linearizados considerando uma onda $\mathrm{P}$ incidente para o caso geral são então dados pelas equações (11), (12) e (13). Nestas expressões as perturbações nos parâmetros elásticos foram separadas em duas componentes

$$
\delta \mathrm{c}_{\mathrm{kmlj}}=\delta \mathrm{c}_{\mathrm{kmlj}}^{\mathrm{iso}}+\delta \mathrm{c}_{\mathrm{kmlj}}^{\mathrm{anis}}
$$

em que $\delta c_{\mathrm{kmlj}}^{\text {iso }}$ é um tensor isotrópico e $\delta \mathrm{c}_{\mathrm{kmlj}}^{\text {anis }}$ define a parte anisotrópica da perturbação nos parâmetros elásticos.

\section{AGRADECIMENTOS}

Os autores agradecem ao apoio do programa FINEP/CNPq/Pronex em Engenharia de reservatórios pela infra-estrutura computacional e ao professor Ivan Psensick pela revisão do manuscrito e discussão deste trabalho.

\section{REFERENCIAS}

Aki, K.\& Richards, P.G, 1980. Quantitative Seismology: Theory and methods, volume 1. W. H. Freeman and Co., San Francisco

Banik, N. C., 1987. An effective anisotropy parameter in transversely isotropic media. Geophysics, 52: 1654-1664.

Castagna, J., 1992. AVO Analysis- Tutorial and Review. In Castagna, J.P. \& Backus, M. M. (Eds). Offset-dependent reflectivity-Theory and practice AVO analysis: Soc. Expl. Geophys. 335 .

Helbig, K., 1994. Foundations of Anisotropy for Exploration Seismics. Handbook of Geophysical Exploration, vol 22, Ed. Pergamon

Hoffman, K. \& Ray, K., 1971. Álgebra Linear. Polígono (ed.).

Hudson, J. A., 1982. Wave speeds and attenuation of elastic waves in material containing cracks. Geophys. J. R. Astr Soc., 64: 133-150.

Musgrave, M. J. P., 1970. Crystal Acoustics. Holden-Day Inc. San Francisco.

Ostrander, W. J., 1984. Plane-wave reflection coefficients for gas sand at non normal angles of incidence. Geophysics, 49: 1637-1648.

Postma, G. W., 1955. Wave propagation in stratified medium. Geophysics, 20(4): 780-806.

Schoenberg, M. \& Douma, J., 1988. Elastic wave propagation in media with parallel fractures and aligned cracks. Geophysics, 56: 1331-1348.

Schoenberg, M. \& Protázio, J.S., 1992. Zoeppritz Rationalized and Generalized to Anisotropy. Journal of Seismic Exploration, 1: 125-144.

Thomsen, 1986. Weak elastic anisotropy. Geophysics, 51: 1954-1966

Thomsen, 1993. Weak elastic anisotropic reflection. In Castagna, J. P. \& Backus, M. M. (Eds.) Offsetdependent reflectivity-Theory and practice AVO analysis: Soc. Exp. Geophys. 103-111.

Vavrycuk, V. \& Psencik, I., 1998. PP-Wave reflection coefficients in weakly anisotropic elastic media. Geophysics, 63 (6):, 2129-2141.

Zillmer, M., Gajewsky, D. \& Kashtan, B. M., 1997. Reflection coefficients for weak anisotropic media. Geophys. J. Internat, 129: 389-398. 